\title{
EFFECT OF CRYSTAL ROTATION ON LIMIT STRAINS OF TEXTURED MATERIALS UNDER EQUI-BIAXIAL TENSION
}

\author{
K. C. CHAN \\ Department of Manufacturing Engineering, The Hong Kong Polytechnic University, \\ Hung Hom, Hong Kong
}

(Received 30 March 1995)

\begin{abstract}
In this paper, a numerical procedure has been proposed to predict limit strains of textured sheets under equi-biaxial tension by means of the MK model and taking into account of crystal rotation. Limit strains have been predicted for three common crystallographic orientations and an annealed aluminium sheet. The effect of crystal rotation on limit strains was shown to be significant. However, no general trend of the effect has been observed. It is argued that the effect is quite complicated and dependent on various factors such as the initial orientation or texture, groove orientation and the initial inhomogeneous factor. The incorporation of texture change in the analysis of sheet metal forming was found essential.
\end{abstract}

KEY WORDS: Limit strains, texture, crystal rotation and sheet metals.

\section{INTRODUCTION}

Since the introduction of the concept of forming limit diagram by Keeler and Backofen (1963) to represent acceptable limit strain during sheet metal forming, it has become a hot research topic for decades and a considerable amount of experimental and theoretical studies have been carried out. Among the earliest theories of the forming limit diagrams. Marciniak and Kuczynski (MK) (1967) have probably proposed the most influential hypothesis for the limit strains prediction. They postulated the existence of an initial imperfection in the sheet which will develop into a groove running in a direction perpendicular to the larger principal stress. The hypothetical groove served as the nucleus of a neck in their model. After then, much theoretical efforts have been made trying to explain and assign the value of the incipient groove depth. Tadros and Mellor (1975) suggested that the incipient grooves may appear during stable plastic flow but that a single groove will not exhibit continuing growth until diffuse necking occurs. They therefore proposed that the MK theory should be applied to the material at the time when diffuse necking sets in. Yamaguchi and Mellor (1976) and Parmar and et al. (1977) have taken explicit account of the influence of grain anisotropy in biaxial stretching. In these models it is assumed that incipient grooves are formed within the roughened surfaces and that, in stretching beyond diffuse instability, strain localization develops within the deepest groove in accordance with the MK model. In the damage mechanics model of the forming limit, 
the formation of groove is related to the internal damage of a sheet (Jalinier and Schmitt, 1982). Preferred orientation of grains which results in plastic anisotropy of a sheet is shown to affect the strain path and hence the limit strain of a sheet in a complicated manner. However, most of these models fail to take into account of texture. Recently, Lin et al. (1991) have based on the MK theories and crystallographic yield surface to study the effect of texture if on limit strain in biaxially stretched steel sheet. Chan and Lee (1990) have shown that the formation of groove is shown to arise from the difference in the thickness strains among grains of different texture components taken along different paths if grains of similar orientation exist as colonies in the sheet. However the effect of crystal rotation on limit strain is less explored. In the present work, a numerical procedure will be first presented for the calculation of the limit strains of textured sheets under equi-biaxial tension by means of the MK model and taking into account of crystal rotation.

\section{Limit Strain Prediction Model}

In the model, a sheet deformed under equi-biaxial tension is considered and the groove is assumed to be perpendicular to the rolling direction as shown in Figure 1. Superscripts $h$ and $g$ are used to identify the properties and parameters associated with the homogeneous and groove zones respectively. The 1-2-3 coordinate system refers to the test axes, in which the $\mathrm{x}$-axis refers to the rolling direction. In equi-biaxial stretching, the stress tensor of the sheet in zone $h$ is denoted by

$$
\sigma^{\mathrm{h}}=\sigma^{\mathrm{h}}\left[\begin{array}{lll}
1 & 0 & 0 \\
0 & 1 & 0 \\
0 & 0 & 0
\end{array}\right]
$$

and the associated strain tensor of equation (1) is denoted by

$$
d \epsilon^{h}=d \epsilon^{h}\left[\begin{array}{ccc}
X^{h} & 0 & 0 \\
0 & 1-X^{h} & 0 \\
0 & 0 & -1
\end{array}\right]
$$

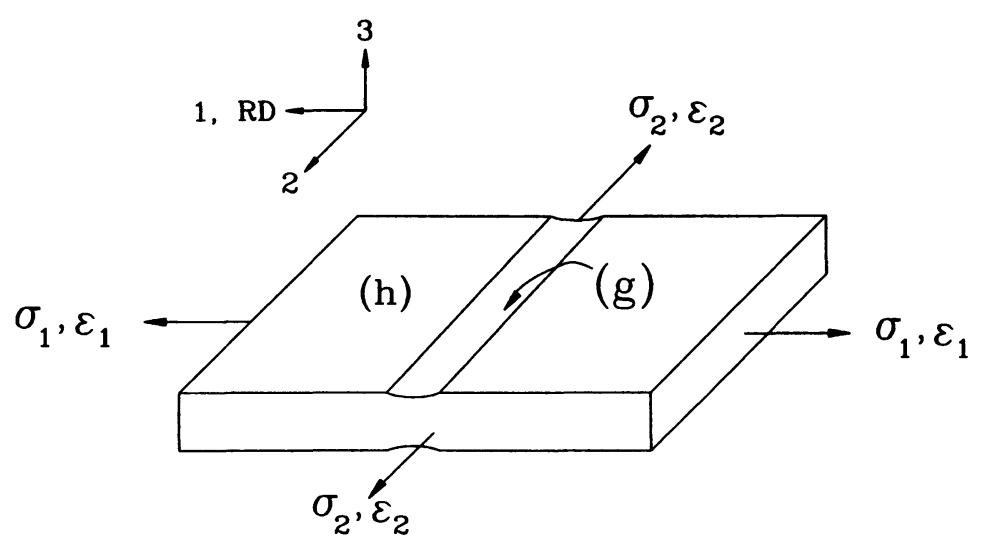

Figure 1 Schematic representation of the geometry of the M-K model. 
In the groove region $\mathrm{g}$, force equilibrium gives

$$
\sigma_{1}^{g}=\sigma_{1}^{h} / f
$$

where $f$ is the inhomogeneity factor and equals $\left(t_{g} / t_{h}\right)$. The groove strain $d \epsilon_{2}^{g}$ is assumed to be the same as the corresponding strain outside the groove:

$$
d \epsilon_{2}^{g}=d \epsilon_{2}^{h}=d \epsilon^{h}\left(1-X^{h}\right)
$$

The strain tensor in the region $\mathrm{g}$ can be written as

where

$$
\epsilon^{g}=d \epsilon^{g}\left[\begin{array}{ccc}
X^{g} & 0 & 0 \\
0 & 1-X^{g} & 0 \\
0 & 0 & -1
\end{array}\right]
$$

$$
d \epsilon^{g}=\left(1-X^{h}\right) d \epsilon^{h} /\left(1-X^{g}\right)
$$

If the groove is parallel to the rolling direction (RD), equations (3), (4) and (6) will be replaced by

respectively.

$$
\begin{gathered}
\sigma_{2}^{g}=\sigma_{2}^{h} / f \\
d \epsilon_{1}^{g}=d \epsilon_{1}^{h}=X^{h} d \epsilon^{h} \\
d \epsilon^{g}=X^{h} d \epsilon^{h} / X^{g}
\end{gathered}
$$

The stress components $\sigma_{\mathrm{ij}}$ and strain components $\epsilon_{\mathrm{ij}}$ are related by the normality principle:

$$
\epsilon_{\mathrm{ij}}=\lambda \partial F\left(\sigma_{i j}\right) / \partial \sigma_{i j}
$$

where $\mathrm{F}\left(\sigma_{i j}\right)$ is the yield function of the sheet metal and $\lambda$ is the proportionality constant. In this paper, an anisotropic continuum yield locus of the Continuum Mechanics of Textured Polycrystals (CMTP) (Montheillet et al. 1985) is adopted and as shown below:

$$
\begin{aligned}
F(S)= & \alpha\left(\left|S_{11}-S_{22}\right|^{\mathrm{n}}+\left|S_{22}-S_{33}\right|^{\mathrm{n}}+\left|S_{33}-S_{11}\right|^{\mathrm{n}}\right) \\
& +2 \beta\left(\left|S_{12}\right|^{\mathrm{n}}+\left|S_{23}\right|^{\mathrm{n}}+\left|S_{31}\right|^{\mathrm{n}}\right)=\left(\sqrt{6} \tau_{\mathrm{c}}\right)^{\mathrm{n}}
\end{aligned}
$$

To calculate $\epsilon^{\mathrm{h}}$, the parameter $\mathrm{x}^{\mathrm{h}}$ in equation (2) is varied until the $\sigma_{i j}$ equals the component of the imposed stress tensor of equation (1). $\epsilon^{\mathrm{g}}$ is also determined by varied $x^{\mathfrak{g}}$ in equation (5) until the force equilibrium requirement of equation (3) or (7) is met. For each small increment of deformation, if crystal rotation is taken into account, new grain orientation can be determined according to the numerical procedures of Chan and Lee (1991). The inhomogeneity factor after each small step of deformation is given by

$$
f=\exp \left(\epsilon_{3}^{g}\right) / \exp \left(\epsilon_{3}^{h}\right)
$$


The procedure of determining $\mathrm{f}$ is repeated until the strain state in the groove region approaches plane strain. The major strain outside the groove region will give the limit strain of the sheet in equi-biaxial tension.

\section{RESULTS AND DISCUSSION}

The limit strains predicted by the present model for three common crystallographic orientations and an annealed aluminium sheet are exhibited. The three orientations considered were $\{100\}<001>,\{112\}<111>,\{123\}<634>$ and the crystallographic texture of the aluminium sheet is quoted from the published crystallite orientation distribution function (Lee and Chan 1991). The volume fraction of the main texture components are as follows $\{100\}<001>(27 \%)+\{112\}<111>(54 \%)+\{123\}<634>$ (19\%). The constants $n, \alpha$ and $\beta$ used in equation (3) are 1.6, 0.46 and 0.51 respectively (Montheillet et al. 1985). Four initial inhomogeneity factor $\mathrm{f}_{\mathrm{i}}$ are chosen and they are $0.96,0.97,0.98,0.99$. In this paper, two different groove orientations (i.e. perpendicular and parallel to the rolling direction) are considered. Tables 1 (a) to (d) show the limit strains of the three orientations and the aluminium sheet predicted by the present model, and the comparison between them and the predicted limit strains without taking crystal rotation into account. It is observed that the predicted limit strains are raised significantly for the orientation $\{100\}<001>$ with the groove perpendicular to the rolling direction when comparing with that without grain rotation.

Table 1 Predicted limit strains $\left(\epsilon_{1}^{*}, \epsilon_{2}^{*}\right)$ for orientations (a) $\{100\}<001>$, (b) $\{112\}<111>$ (c) $\{123\}$ $<634>$ and (d) an aluminium sheet.

(a) $\{100\}<001>$

\begin{tabular}{l|cc|cc|cc|rr}
\hline & \multicolumn{4}{c|}{ With Crystal Rotation } & \multicolumn{3}{c}{ Without Crystal Rotation } \\
\cline { 2 - 9 } & \multicolumn{2}{|c|}{ Groove $\perp$ to $R D$} & \multicolumn{2}{c|}{ Groove // to $R D$} & Groove $\perp$ to $R D$ & Groove // to $R D$ \\
\cline { 2 - 9 }$f_{i}$ & $\epsilon_{I}^{*}$ & $\epsilon_{2}^{*}$ & $\epsilon_{I}^{*}$ & $\epsilon_{2}^{*}$ & $\epsilon_{I}^{*}$ & $\epsilon_{2}^{*}$ & $\epsilon_{I}^{*}$ & $\epsilon_{2}^{*}$ \\
\hline 0.96 & 0.1868 & 0.1632 & 0.1631 & 0.1469 & 0.1647 & 0.1652 & 0.1647 & 0.1653 \\
0.97 & 0.2238 & 0.1862 & 0.1868 & 0.1632 & 0.1897 & 0.1903 & 0.1897 & 0.1903 \\
0.98 & $*$ & $*$ & 0.2428 & 0.1972 & 0.2196 & 0.2204 & 0.2196 & 0.2204 \\
0.99 & $*$ & $*$ & 0.3577 & 0.2623 & 0.2646 & 0.2654 & 0.2697 & 0.2704 \\
\hline
\end{tabular}

(b) $\{112\}<111>$

\begin{tabular}{|c|c|c|c|c|c|c|c|c|}
\hline \multirow[b]{3}{*}{$f_{i}$} & \multicolumn{4}{|c|}{ With Crystal Rotation } & \multicolumn{4}{|c|}{ Without Crystal Rotation } \\
\hline & \multicolumn{2}{|c|}{ Groove $\perp$ to $R D$} & \multicolumn{2}{|c|}{ Groove // to $R D$} & \multicolumn{2}{|c|}{ Groove $\perp$ to $R D$} & \multicolumn{2}{|c|}{ Groove // to $R D$} \\
\hline & $\epsilon_{I}^{*}$ & $\epsilon_{2}^{*}$ & $\epsilon_{I}^{*}$ & $\epsilon_{2}^{*}$ & $\epsilon_{I}^{*}$ & $\epsilon_{2}^{*}$ & $\epsilon_{I}^{*}$ & $\epsilon_{2}^{*}$ \\
\hline 0.96 & 0.3226 & 0.4874 & 0.2082 & 0.3418 & $* *$ & $* *$ & 0.1806 & 0.3194 \\
\hline 0.97 & 0.3523 & 0.5177 & 0.2372 & 0.3828 & ** & ** & 0.2167 & 0.3833 \\
\hline 0.98 & 0.3892 & 0.5508 & 0.2808 & 0.4392 & $* *$ & $* *$ & 0.2673 & 0.4727 \\
\hline 0.99 & 0.4529 & 0.5971 & 0.3678 & 0.5322 & ** & $* *$ & 0.3539 & 0.6261 \\
\hline
\end{tabular}


(c) $\{123\}\langle 634\rangle$

\begin{tabular}{l|cc|cc|cc|cc}
\hline & \multicolumn{4}{|c|}{ With Crystal Rotation } & \multicolumn{4}{c}{ Without Crystal Rotation } \\
\cline { 2 - 10 } & \multicolumn{2}{|c|}{ Groove $\perp$ to $R D$} & \multicolumn{2}{c|}{ Groove // to $R D$} & \multicolumn{2}{c}{ Groove $\perp$ to $R D$} & Groove // to $R D$ \\
\cline { 2 - 10 }$f_{i}$ & $\epsilon_{1}^{*}$ & $\epsilon_{2}^{*}$ & $\epsilon_{1}^{*}$ & $\epsilon_{2}^{*}$ & $\epsilon_{1}^{*}$ & $\epsilon_{2}^{*}$ & $\epsilon_{1}^{*}$ & $\epsilon_{2}^{*}$ \\
\hline 0.96 & 0.1770 & 0.1430 & 0.6257 & 0.3143 & 0.2839 & 0.3061 & 0.2743 & 0.2957 \\
0.97 & 0.2093 & 0.1607 & 0.8298 & 0.3702 & 0.3417 & 0.3683 & 0.3224 & 0.3476 \\
0.98 & 0.2429 & 0.1771 & $* *$ & $* *$ & 0.4283 & 0.4617 & 0.3994 & 0.4306 \\
0.99 & 0.3126 & 0.2074 & $* *$ & $* *$ & 0.5727 & 0.6173 & 0.5294 & 0.5707 \\
\hline
\end{tabular}

(d) Aluminium sheet

\begin{tabular}{|c|c|c|c|c|c|c|c|c|}
\hline \multirow[b]{3}{*}{$f_{i}$} & \multicolumn{4}{|c|}{ With Crystal Rotation. } & \multicolumn{4}{|c|}{ Without Crystal Rotation } \\
\hline & \multicolumn{2}{|c|}{ Groove $\perp$ to $R D$} & \multicolumn{2}{|c|}{ Groove // to RD } & \multicolumn{2}{|c|}{ Groove $\perp$ to $R D$} & \multicolumn{2}{|c|}{ Groove // to $R D$} \\
\hline & $\epsilon_{1}^{*}$ & $\epsilon_{2}^{*}$ & $\epsilon_{I}^{*}$ & $\epsilon_{2}^{*}$ & $\epsilon_{I}^{*}$ & $\epsilon_{2}^{*}$ & $\epsilon_{l}^{*}$ & $\epsilon_{2}^{*}$ \\
\hline 0.96 & 0.2612 & 0.3388 & 0.2075 & 0.2825 & 0.3857 & 0.5943 & 0.1850 & 0.2850 \\
\hline 0.97 & 0.2923 & 0.3677 & 0.2416 & 0.3189 & 0.4604 & 0.7096 & 0.2204 & 0.3396 \\
\hline 0.98 & 0.3361 & 0.4039 & 0.2923 & 0.3677 & 0.4722 & 0.7278 & 0.2715 & 0.4185 \\
\hline 0.99 & 0.4186 & 0.4614 & 0.3884 & 0.4416 & 0.5727 & 0.6173 & 0.3621 & 0.5579 \\
\hline
\end{tabular}

* unable to form localized necking

** too large to report

Nevertheless, the limit strains are lowered dramatically for the orientation $\{112\}<111>$ with the same groove orientation. Whereas, for the orientation $\{123\}<634>$, different groove directions result in significantly different effect on the limit strains. While the limit strains are raised tremendously when the groove is parallel to the rolling direction, they are lowered when the groove is perpendicular to the rolling direction. Similar but less significant effect as that of the orientation $\{123\}<634>$ is observed for the aluminium sheet. Grain rotation is shown to affect limit strains of a sheet in a complicated manner; no general trend is observed.

Minimum limit strains among the two groove orientations are also plotted against the initial inhomogeneity factors as shown in Figures 2 (a) to (d). The predicted limit strains with the consideration of crystal rotation are found to be higher for the orientation $\{100\}<001>$ and $\{123\}<634>$ and the aluminium sheet. Whereas much lower predicted limit strains are observed for the $\{112\}<111>$. No simple trend of the effect of grain rotation on limit strain can be observed. In fact, grain rotation as well as initial texture are related to strain paths of a sheet as reported by Chan and Lee (1990). In their works, they showed that curvilinear paths are often found for most orientations in biaxial tension when grain rotation is taken into account, and that the effect of grain rotation on strain path depends on initial texture. On the other hand, strain paths have already been reported to relate to limit strains (Korhonen, 1978). It is then argued that the effect of grain rotation on limit strain is quite complicated and dependent on the initial orientation or texture, groove orientation and the initial inhomogeneous factor. Further theoretical works have to be carried out to consider more orientations and materials. 
(a) $\{100\}<001>$

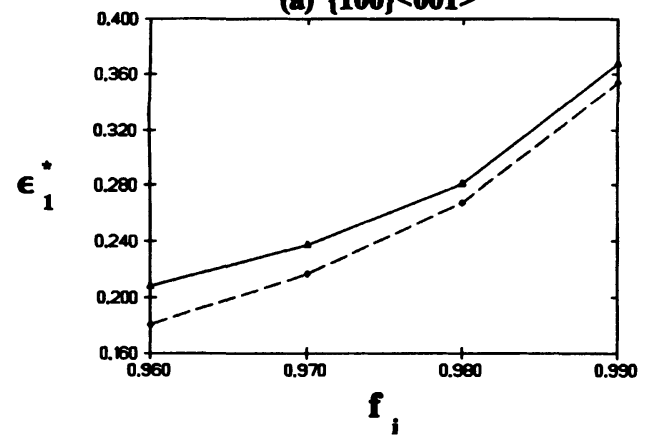

(c) $\{123\}<634>$

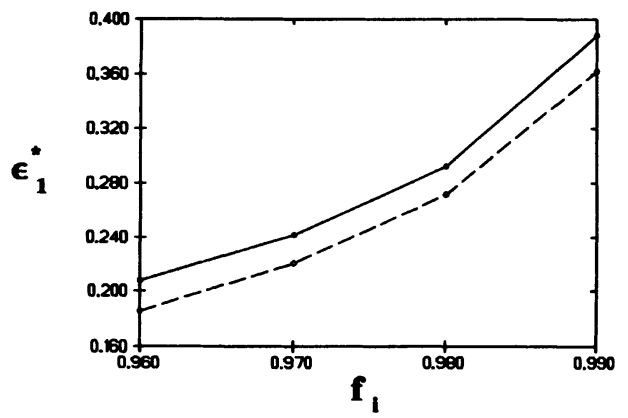

(b) $\{112\}<111>$

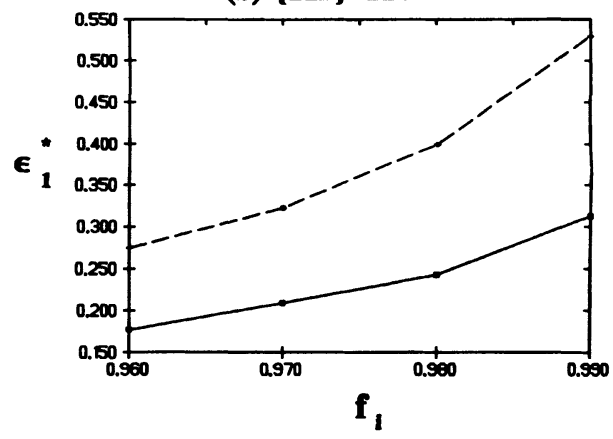

(d) Aluminium sheet

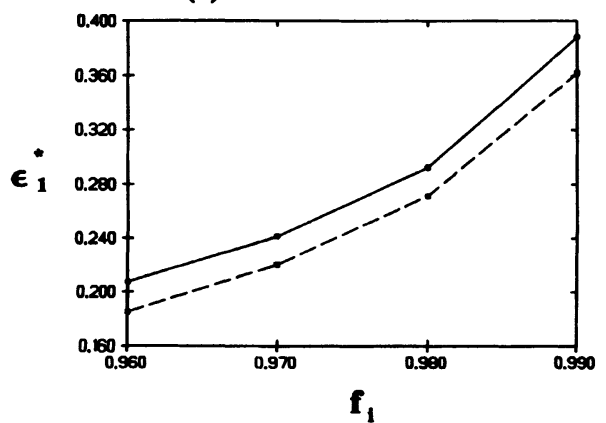

Figure 2 Predicted minimum limit strains for orientations (a) $\{100\}<001>$, (b) $\{112\}<111>$, (c) $\{123\}<634>$ and (d) an aluminium sheet. The limit strains with and without consideration of crystal rotation are represented by - and - - - respectively.

\section{CONCLUSION}

In this work, a numerical procedure based on the MK model and taking crystal rotation into account has been proposed to predict the limit strains in sheet metal forming. It is found that the effect of crystal rotation on limit strains is significant. The incorporation of texture change in the analysis of sheet metal forming is hence essential. More accurate predictions might be obtained if the model can incorporate other theories in assigning initial inhomogeneous factor and a more sophisticated model of crystal rotation.

\section{References}

Chan, K. C. and Lee, W. B. (1990). Int. J. Mech. Sci., 32, 497.

Jalinier, J. M. and Schmitt, J. H. (1982). Acta Metall. 30, 1789.

Keeler, S. P. and Backofen, A. (1963). Met. Trans. A, 56, 25.

Korhonen, A. S. (1978). J. Engg. Mat. Tech., 100, 303.

Lee, W. B. and Chan, K. C., Proceedings of the 3rd International Conference on Technology of Plasticity, Kyoto, Japan, July 1990, pp. 1285-1292.

Lee, W. B. and Chan, K. C. (1991). Texture and Microstructures, 14, 1221.

Lin, D. W., Daniel, D. and Jonas, J. J. (1991). Metall. Trans. A, 22A, 2069.

Marciniak, Z. and Kuczynski, K. (1967). Int. J. Mech. Sci., 9, 609. 
Montheillet, F, Gilormini, P. and Jonas, J. J. (1985). Acta Metall., 33, 705.

Parmar, A., Mellor, P. B. and Chakrabarty, J. (1977). Int. J. Mech. Sci. 19, 389.

Tadros, A. K. and Mellor, P. B. (1975). Int. J. Mech. Sci. 17, 203.

Yamaguchi, K. and Mellor, P. B. (1976). Int. J. Mech. Sci. 18, 85. 